\title{
Zebrafish Tie-2 shares a redundant role with Tie-1 in heart development and regulates vessel integrity
}

\author{
Evisa Gjini ${ }^{1}$, Liesbeth H. Hekking ${ }^{2}$, Axel Küchler ${ }^{3, *}$, Pipsa Saharinen ${ }^{4}$, Erno Wienholds ${ }^{1}$, Jan-Andries Post ${ }^{2}$, Kari Alitalo ${ }^{4}$ and \\ Stefan Schulte-Merker ${ }^{1, \neq}$
}

\begin{abstract}
SUMMARY
Tie-2 is a member of the receptor tyrosine kinase family and is required for vascular remodeling and maintenance of mammalian vessel integrity. A number of mutations in the human TIE2 gene have been identified in patients suffering from cutaneomucosal venous malformations and ventricular septal defects. How exactly Tie-2 signaling pathways play different roles in both vascular development and vascular stability is unknown. We have generated a zebrafish line carrying a stop mutation in the kinase domain of the Tie-2 receptor. Mutant embryos lack Tie- 2 protein, but do not display any defect in heart and vessel development. Simultaneous loss of Tie-1 and Tie-2, however, leads to a cardiac phenotype. Our study shows that Tie-1 and Tie-2 are not required for early heart development, yet they have redundant roles for the maintenance of endocardial-myocardial connection in later stages. Tie-2 and its ligand Angiopoietin-1 have also been reported to play an important role in vessel stability. We used atorvastatin and simvastatin, drugs that cause bleeding in wild-type zebrafish larvae, to challenge vessel stability in tie-2 mutants. Interestingly, recent clinical studies have reported hemorrhagic stroke as a side effect of atorvastatin treatment. Exposure of embryos to statins revealed that tie-2 mutants are significantly protected from statin-induced bleeding. Furthermore, tie-2 mutants became less resistant to bleeding after VE-cadherin knockdown. Taken together, these data show that atorvastatin affects vessel stability through Tie-2, and that VE-cadherin and Tie-2 act in concert to allow vessel remodeling while playing a role in vessel stability. Our study introduces an additional vertebrate model to study in vivo the function of Tie- 2 in development and disease.
\end{abstract}

\section{INTRODUCTION}

Tie-1 and Tie-2 constitute the Tie family of tyrosine kinase receptors, and both are expressed mainly in endothelial cells (ECs) and early hematopoietic cells (Sato et al., 1993; Iwama et al., 1993; Dumont et al., 1995; Yano et al., 1997). Gene-targeting experiments for Tie-1 (Sato et al., 1995; Puri et al., 1995) and Tie-2 (Sato et al., 1995; Puri et al., 1995; Patan, 1998; Dumont et al., 1994; Puri et al., 1999) result in embryonic lethality in mice, indicating that both genes are required for normal embryonic development. Tie- $2^{-/-}$ mice die at embryonic day 9.5 (E9.5)-E12.5 owing to both a failure to remodel the primary capillary plexus and to cardiac defects caused by poor association between endothelial cells and the underlying matrix and to lack of myocardial trabecular projections (Dumont et al., 1994; Puri et al., 1999). Tie-1 homozygous mutants

\footnotetext{
${ }^{1}$ Hubrecht Institute-KNAW and University Medical Centre, Uppsalalaan 8, 3584 CT Utrecht, The Netherlands

${ }^{2}$ Cellular Architecture and Dynamics, Utrecht University, 3508 TC Utrecht, The Netherlands

${ }^{3}$ Exelixis Inc., 170 Harbor Way, South San Francisco, CA 94083, USA

${ }^{4}$ Molecular/Cancer Biology Laboratory and Ludwig Institute for Cancer Research, Biomedicum Helsinki, University of Helsinki, Haartmaninkatu 8, 00014 Helsinki, Finland

*Present address: Institute of Pathology, Rikshospitalet University Hospital, 0424 Oslo, Norway

${ }^{\ddagger}$ Author for correspondence (s.schulte@hubrecht.eu)

Received 1 December 2009; Accepted 13 September 2010

(c) 2011. Published by The Company of Biologists Ltd

This is an Open Access article distributed under the terms of the Creative Commons Attribution Non-Commercial Share Alike License (http://creativecommons.org/licenses/by-nc-sa/3.0), which permits unrestricted non-commercial use, distribution and reproduction in any medium provided that the original work is properly cited and all further distributions of the work or adaptation are subject to the same Creative Commons License terms
}

are embryonic or neonatal lethal, and display a phenotype characterized by hemorrhages, abnormal vascular endothelial cell development and edema (Sato et al., 1995; Puri et al., 1995). Although both $\mathrm{Tie}^{-1^{-/}}$and Tie-2 ${ }^{-/-}$mice exhibit vascular defects, studies conducted making use of mosaic analysis in double Tie-1 and Tie-2 mutants have shown that both genes are dispensable for the processes of early angiogenic sprout formation and perivascular cell recruitment in extraembryonic and embryonic tissues (Puri et al., 1999). Therefore, the vascular deficiencies are secondary to the endocardial defects in both Tie- $1^{-/}$and Tie- $2^{-/-}$knockouts. Tie1 and Tie-2 interact during cardiac development (Puri et al., 1999). Embryos lacking both copies of Tie-1 but only one copy of Tie-2 showed heart failure indicated by pericardium edema and poor maintenance of the atrial and ventricular endocardium (Puri et al., 1999). Mutations in the human TIE- 2 receptor have been identified in patients with cutaneomucosal venous malformations (VMCM; OMIM \#600195), characterized by dilated blood vessels primarily at the mucosa (Vikkula et al., 1996; Wouters et al., 2010). Consistent with the function of murine Tie-2 in vessel and cardiac development, VMCM patients have a relative lack of mural cells in affected regions of the endothelium and some have ventricular septal defects (VSDs) (Vikkula et al., 1996; Wouters et al., 2010).

Angiopoietins 1-4 (Ang-1-Ang-4) serve as ligands for the Tie2 receptor, with Ang-1 and Ang-2 being the most extensively studied to date (Yancopoulos et al., 2000; Geva and Jaffe, 2000). Ang-1 acts as an agonist of Tie-2, whereas Ang-2 can act either as an agonist or an antagonist, depending on the cell type and the microenvironment (Maisonpierre et al., 1997; Teichert-Kuliszewska et al., 2001; Saharinen et al., 2005). Ligands for Tie-1 have not been identified yet. However, it has been reported that Ang-1 stimulates the activation of Tie- 1 in the presence of Tie-2 (Suri et al., 1996). 
In endothelial cells, binding of Ang-1 to Tie-2 promotes angiogenesis, vessel stabilization and pericyte recruitment (Suri et al., 1998; Thurston et al., 1999; Thurston et al., 2000; Fiedler et al., 2004), whereas Ang-2 provides autocrine vessel destabilization signals by inhibiting Tie-2 signaling (Fiedler et al., 2006; Gamble et al., 2007). This leads to pericyte detachment and disrupted extracellular matrix (ECM) interactions, making the endothelial cells more responsive to a variety of stimuli (Thurston et al., 2000). Today, no mutations in Ang-1 or Ang-2 have been identified in VMCM patients, possibly suggesting a ligand-independent TIE-2 function in this malignancy (Wouters et al., 2010).

The current notion is that Ang-1-Tie-2 signaling inhibits endothelial permeability in mature vessels (Fiedler et al., 2004), whereas Ang-2-Tie-2 signaling destabilizes the vasculature (Fiedler et al., 2006; Gamble et al., 2007). The vessel stability induced by Ang-1 depends on tightening of endothelial cell-cell junctions as well as on increased association of the endothelium with pericytes and smooth muscle cells (Mammoto et al., 2007). Ang-1 is shown to signal via Rho family GTPases that organize the cytoskeleton accordingly to allow stable cell-cell junction organization, thereby enhancing the barrier function of the endothelium (Mammoto et al., 2007). More specifically, Ang-1 induces activation of Rac1 and inhibition of RhoA (Mammoto et al., 2007). RhoA promotes formation of actin stress fibers, which increase centripetal tension throughout the cytoskeleton, whereas Rac1 counterbalances this by regulating the maintenance of adherent and tight junctions (Mammoto et al., 2007). In 2007, Gavard et al. reported that Ang-1 prevents VEGF-induced vessel permeability by activation of $\mathrm{mDia}$ via RhoA, resulting in sequestering of Src through $\mathrm{mDia}$. This in turn inhibits VE-cadherin internalization and thus leads to stabilized endothelial cell-cell junctions and reduced endothelial permeability (Gavard et al., 2007). Other recent studies (Saharinen et al., 2008; Fukuhara et al., 2008) have elucidated the contextdependent effects of Ang-1-Tie-2 signaling: in resting ECs, Tie-2 translocates to the cell junctions in a ligand-dependent manner and assembles a homotypic in-trans-complex with Tie- 2 from neighboring cells. In conjunction with Tie-1 and the phosphatase VE-PTP, a complex is formed, which activates Akt serine kinase and eNOS signaling. In motile endothelial cells, however, Tie-2 is translocated to focal adhesions, where it phosphorylates the Dok$\mathrm{R}$ docking protein and affects the migratory behavior of the cell (Saharinen et al., 2008; Fukuhara et al., 2008).

The Tie-1 and Tie-2 homologs in zebrafish show a high degree of sequence similarity with their murine and human orthologs (Lyons et al., 1998). At 24 hours post-fertilization (hpf), both Tie genes are expressed in an endothelium-specific manner in the head vasculature, dorsal aorta and posterior cardinal vein (PCV), and also in the endocardium. tie-1, but not tie-2, is also expressed in intersegmental vessels (Lyons et al., 1998). Even though the importance of the Tie receptors for endothelial biology has long been recognized, a functional analysis of the genes encoding these proteins in an in vivo system different from the mouse has been missing. Here, we show that Tie-1 and Tie- 2 have redundant roles in heart development in zebrafish, and that loss of Tie- 2 signaling protects zebrafish from atorvastatin- and simvastatin-induced bleeding. Therefore, the absence of a heart phenotype in the tie-2 mutant fish allows studying the role of Tie- 2 at later stages of development and disease.

\section{RESULTS}

\section{tie-2 hu1667 encodes for a premature stop codon}

A mutant in the zebrafish tie-2 gene was generated by TILLING (Wienholds et al., 2002). The identified allele of the tie-2 mutant, tie-2 ${ }^{h u 1667}$, encodes Tie-2Q982stop, in which the codon CAG [glutamine (Q)] is mutated to a TAG (stop) codon (Fig. 1A). The glutamine at position 982 in the Tie-2 protein is highly conserved between species (Lyons et al., 1998) and it is positioned in the kinase domain of the Tie-2 receptor upstream of Y1094 and Y1105, residues that are predicted to be crucial for auto-phosphorylation. Furthermore, we observed a strong decrease in tie-2 mRNA levels in mutants compared with wild-type siblings at $24 \mathrm{hpf}$ (Fig. 1B) and a complete loss at $48 \mathrm{hpf}$ (Fig. 1B), most probably due to nonsense-mediated decay. We also performed immunohistochemistry with an antibody that recognizes the EGF-like domain in the extracellular part of Tie-2. Whereas, in wild-type fish, Tie-2 protein is expressed strongly in the PCV, Tie-2 protein expression is completely lost in tie-2 mutants (Fig. 1C).

\section{Tie- 1 and Tie-2 have a redundant role in the maintenance of endocardial-myocardial connection during heart development}

Homozygous tie-2 mutants are viable and grow to adulthood. In order to assess whether lack of a phenotype in tie-2 mutants is due to redundancy between Tie-1 and Tie-2, we injected different doses of a tie-1 morpholino (splice-donor site; sd-tie-1 MO; see supplementary material Fig. S1A,B) into embryos from a cross of two tie-2 heterozygous fish in the $\operatorname{tg}(f l i 1: E G F P) y 1$ background (Lawson et al., 2002) (Fig. 2Ai-ii). No morphological, vascular nor cardiac defects were apparent at 24 and $48 \mathrm{hpf}$. At day 3, however, we observed phenotypes in 33\% of the embryos injected with $3 \mathrm{ng}$ sd-tie-1 MO (98 out of 300), 43\% of the embryos injected with 6 ng (137 out of 320 ) and 52\% of the embryos injected with $8 \mathrm{ng}$ (165 out of 317). Two phenotypes were apparent: $80 \%$ of the phenotypic embryos had blood accumulation in the pericardial sac (Fig. 2Aiii), and $20 \%$ had cardiac edema and arrested blood circulation (Fig. 2Aiv). Genotyping of affected and non-affected embryos showed that, at a dose of $3 \mathrm{ng} / \mathrm{embryo}$ sd-tie-1 MO, 11\% (8 out of 73) of the homozygous wild-type embryos, $27 \%$ (42 out of 153 ) of the tie2 heterozygous and $65 \%$ (48 out of 74 ) of the tie- 2 homozygous mutants displayed blood accumulation in the pericardial sac, and cardiac edema. In embryos injected with the highest $s d$-tie-1 MO dose $(8 \mathrm{ng})$, the number of phenotypic embryos related to the genotyping results as follows: homozygous wild type, 31\% (25 out of 81 ); tie-2 heterozygous mutants, $50 \%$ (80 out of 160 ); and tie-2 homozygous mutants, $80 \%$ (66 out of 83 ) (supplementary material Fig. S1C). The phenotypes were never observed in the uninjected embryos $(n=250)$ from a cross of two tie-2 heterozygotes, which were sorted and genotyped using the same criteria as for the injected group. Statistical analysis showed that the correlation of genotype and phenotype was significant.

To further investigate the pericardial blood accumulation phenotype, we examined transverse sections in the heart region of ten tie-2 mutants (Fig. 2Bi-iii) and of tie-2 mutants injected with the sd-tie-1 MO (Fig. 2Ciiii). Uninjected siblings and uninjected tie-2 mutants exhibited normal cardiac morphology, with the endocardium and myocardium layers closely abutting within the atrium and ventricular zones (Fig. 2Bii,iii). However, in tie-2 mutants injected with $s d$-tie-1 MO, we observed a detachment of 

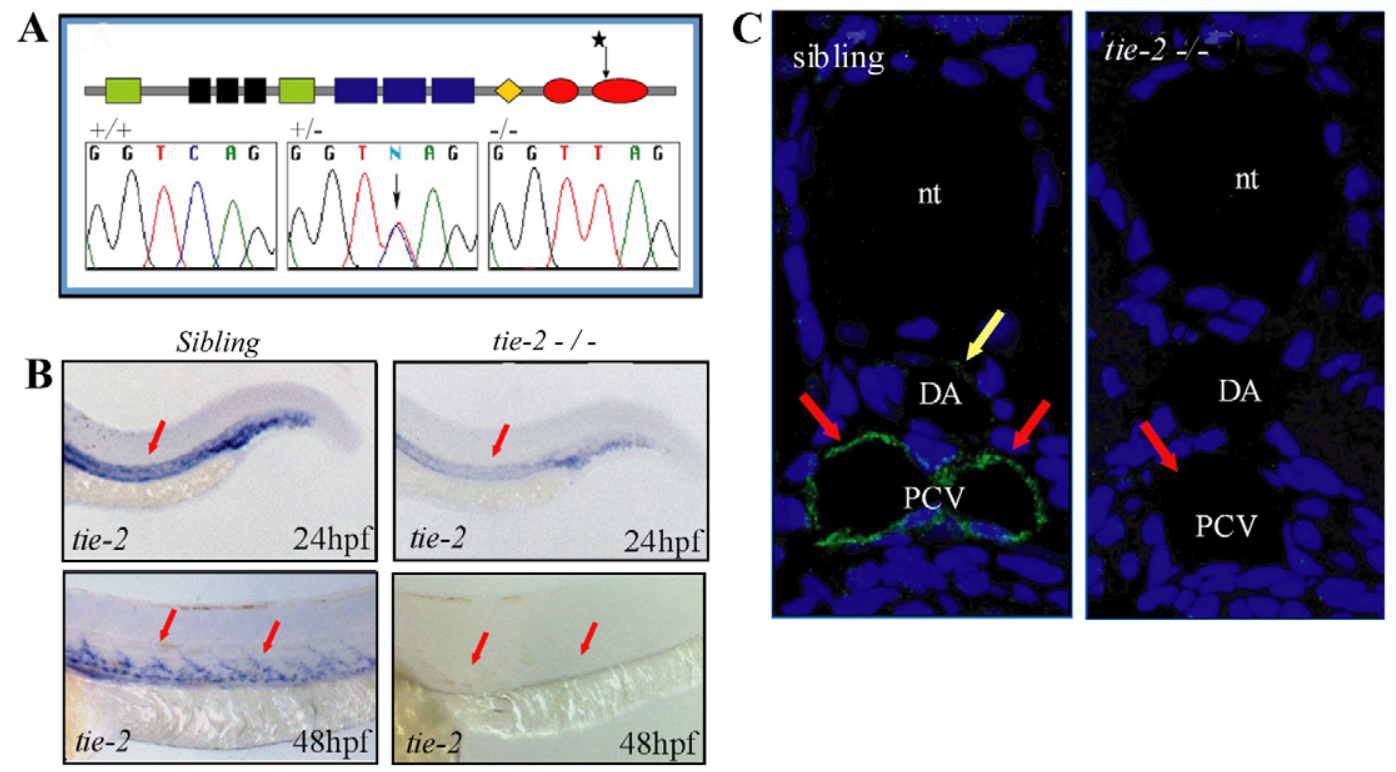

Fig. 1. A mutation in the kinase domain of tie-2 leads to loss of Tie-2 protein. (A) Schematic of the Tie-2 protein domains. Green, black and blue boxes represent the Ig-like, EGF-like and FN-III protein domains, respectively. The orange diamond represents the transmembrane region and the two red ovals

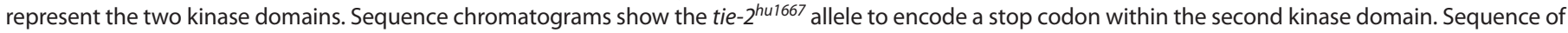
the tie-2 ${ }^{\text {hu1667 }}$ allele: CAG (Q982) is changed into a TAG (STOP) codon (mutation indicated by a star). (B) At 24 hpf, tie-2 mRNA expression levels are significantly

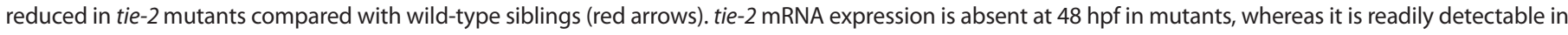
wild-type siblings (red arrows). (C) In wild-type fish, Tie-2 protein is expressed in the PCV at 48 hpf. Tie- 2 protein expression is completely undetectable in tie-2 mutants. Yellow arrow shows Tie-2 protein expression in the dorsal aorta (DA); red arrows show Tie-2 protein expression in the PCV. nt, notochord.

the endocardium from the myocardium, with endothelial cells exhibiting thin cytoplasmic protrusions attached to the myocardium in the equivalent regions (Fig. 2Cii,iii). In addition, we performed electron microscopy to better understand, at subcellular resolution, how the endocardium and myocardium layers are affected in these fish. In tie-2 mutants injected with $s d$ tie-1 MO ( $n=2$ ) (Fig. 2Civ), the contact between the membranes of the cardiomyocytes and endothelial cells was disturbed, as was the cardiac jelly. In tie-2 mutants $(n=2)$ (Fig. 2Biv) and wild-type fish injected with sd-tie-1 MO (data not shown), the membranes of cardiomyocytes and endothelial cells were in close contact. To investigate whether the phenotype that we observed in tie-2 mutants injected with sd-tie-1 MO is a result of loss of circulation, we analyzed the architecture of endocardium and myocardium in plc $g^{-/-}$mutants, which lack blood flow (Bussmann et al., 2010) (supplementary material Fig. S2). Our data show that $\mathrm{plcg}^{-/}$ mutants show no defects in endocardial-myocardial contacts. These results demonstrate a redundant and specific role for Tie-1 and Tie- 2 in maintaining the integrity of endocardial-myocardial connectivity.

\section{tie-1 knockdown in tie-2 mutants does not affect heart chamber differentiation and heart cushion formation}

To investigate whether early heart developmental processes such as heart chamber specification and heart cushion formation were affected in fish lacking both tie-1 and tie-2 signaling, we performed whole-mount in situ hybridization to check for expression of atrial natriuretic factor (nppa) and hyaluronan synthase 2 (has2) in uninjected wild-type embryos and tie-2 mutants, or embryos injected with sd-tie-1 MO (Fig. 3).
Changes in the expression of the chamber-specific gene nppa were not observed in wild-type embryos (Fig. 3Ai) compared with tie-2 mutants (Fig. 3Aii). In addition, we did not observe any differences in nppa expression after tie-1 knockdown in wild-type fish (Fig. 3Aiii) or tie-2 mutants (Fig. 3Aiv). The atrioventricular boundary formed correctly during early steps of heart valve formation and expressed the has 2 marker in wild-type embryos (Fig. 3Bi), tie-2 mutants (Fig. 3Bii), wild-type fish injected with $s d-$ tie-1 MO (Fig. 3Biii) and tie-2 mutants injected with sd-tie-1 MO (Fig. 3Biv).

Embryos lacking both Tie-1 and Tie-2 showed no defects in heart looping. Moreover, knockdown of tie- 1 alone or in the tie- 2 mutant background did not affect heart chamber specification and heart valve formation in zebrafish. Therefore, we conclude that neither the knockdown of tie- 1 alone nor the combined knockdown of tie-1 and tie-2 affects heart development up to $48 \mathrm{hpf}$.

Consistent with these findings, we observed that morpholinomediated knockdown of angiopoietin $1 a$ and angiopoietin $2 a$ did not affect heart and blood vasculature in zebrafish (supplementary material Figs S3 and S4). Whereas angiopoietin 1 and angiopoietin 2 are duplicated (supplementary material Fig. S5A), reverse transcriptase PCR (RT-PCR) data showed that only angiopoietin $1 a$ and angiopoietin $2 a$ are expressed during early development (data not shown).

\section{Loss of Tie-2 protects from statin-induced head hemorrhages}

Tie-2 and its ligands have been shown to play a role in vasculogenesis (Patan, 1998; Dumont et al., 1994), angiogenesis (Saharinen et al., 2005; Suri et al., 1996; Gale et al., 2002) and lymphangiogenesis (Gale et al., 2002). We therefore examined, using 

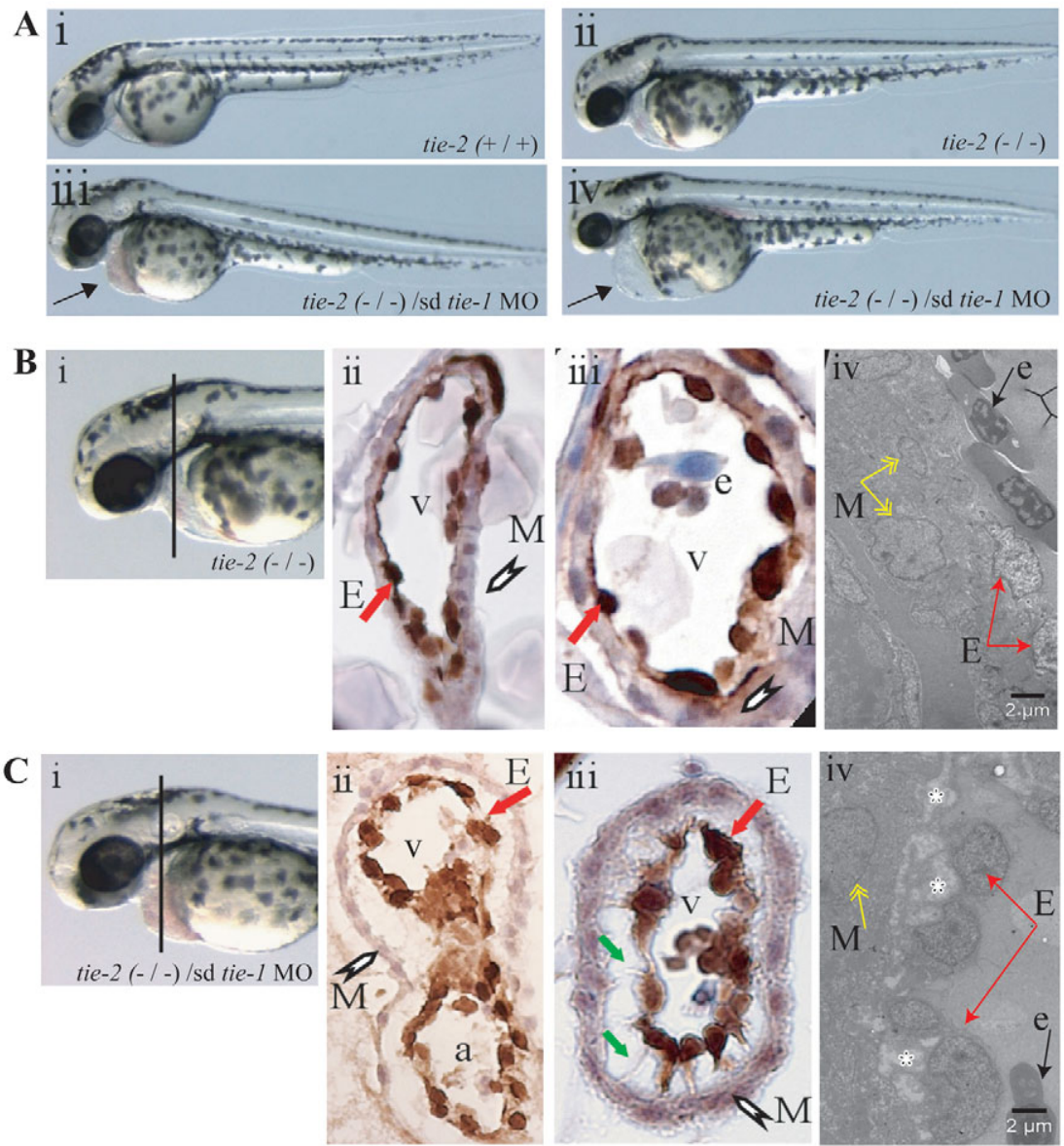

Fig. 2. Loss of tie-1 function in tie-2 mutants results in severe defects in endocardial-myocardial contact. (A) Wild type (i) and tie-2 mutants (ii) show no cardiac defects, whereas tie-1 morpholino knockdown in tie-2 mutants (iii,iv) leads to blood accumulation (arrow in iii) in the pericardial sac or to cardiac edema (arrow in iv) and arrested blood circulation (iv). (B) Immunohistochemistry using an anti-GFP antibody on heart cross-sections from 3-dpf tie-2 mutants in tg(fli1:EGFP)y 1 background (i-iii) reveals no defects in myocardial (white arrowhead) and endocardial (red arrow) contact. (Biv) See below. (C) Immunohistochemistry using an anti-GFP antibody on heart cross-sections from 3-dpf tie-2 mutants in the $\operatorname{tg}(f l i 1: E G F P) y 1$ background injected with sd-tie-1 $\mathrm{MO}$ (i-iii) reveals defects in myocardial (white arrowhead) and endocardial (red arrow) contacts. Endothelial cells exhibit thin cytoplasmic protrusions (green arrows) attached to the myocardium. (Biv,Civ) EM studies demonstrate defects (depicted by white asterisks) in endocardial (red arrows) and myocardial (yellow arrows) lining in tie-2 mutants injected with sd-tie-1 MO (Civ) but not in control tie-2 mutants (Biv). Black arrow depicts an erythrocyte. $M$, myocardium; $\mathrm{E}$, endocardium; $\mathrm{V}$, ventricle; e, erythrocyte; a, atrium. a number of different transgenic lines such as $\operatorname{tg}(f l i 1: E G F P) y 1$ and $\operatorname{tg}(k d r-l: E G F P)$ (Jin et al., 2005), both blood and lymphatic vasculature in tie-2 mutants at 24, 48 and $120 \mathrm{hpf}$. This revealed normal development of head and trunk vasculature (Fig. 4Ai,ii), including normal lymphatic vasculature (Fig. 4Aiii,iv). We also performed sub-epidermal injection tests for lymphatic function: dye that was injected into the trunk of a 5-day-old tie-2 mutant $(n=10)$ was taken up by the thoracic duct, as in the wild-type situation ( $n=10)$ (Fig. 4Bi), showing that the lymphatic vasculature is functional in mutants (Fig. 4Bii).

Because Tie-2 signaling has also been implied in vascular permeability (Thurston et al., 1999; Thurston et al., 2000), we were looking for ways to challenge vessel stability in tie-2 mutants. Because human patients deficient in CCM1 protein function have been shown to suffer from brain hemorrhaging and because tie-2 expression has been reported to be elevated in $c \mathrm{~cm}-1$ mutants (Jin et al., 2007), we created double mutants in tie-2 and ccm-1 (Hogan et al., 2008), but did not observe any changes in phenotypic strength when compared with $\mathrm{ccm}-1$ mutants (data not shown). An alternative approach we took was to expose embryos to atorvastatin, a drug that had been noticed to induce intracranial hemorrhages in wild-type fish (Küchler, 2004). Offspring of two heterozygous mutant carriers were exposed to different concentrations of atorvastatin $(50,150,300,500$ and $1000 \mathrm{nM})$ at $6 \mathrm{hpf}$ for 48 hours ( $n=60$ per group in three separate experiments). Hemorrhages were identified through o-dianizidine staining, and genotyping for the tie-2 mutation was performed subsequently. We observed that the number of hemorrhagic wild-type fish (Fig. 5Ai) was significantly higher than the number of hemorrhagic tie-2 mutants (Fig. 5Aii) at every atorvastatin concentration tested. For example, at $150 \mathrm{nM}$ atorvastatin, $50 \%$ of the wild type bled, whereas only $5 \%$ of the mutants showed bleedings.

To determine whether the observed protective phenotype was atorvastatin-specific, we also exposed fish to simvastatin, another statin drug. Offspring of two heterozygous mutant carriers were exposed to different concentrations of simvastatin $(5,10,25,50$ and $100 \mathrm{nM}$ ) at $6 \mathrm{hpf}$ for 48 hours ( $n=60$ per group in three separate experiments). As in the atorvastatin exposure experiment, the number of hemorrhagic wild-type fish after exposure to simvastatin (Fig. 5Aiii) was significantly higher than the number of hemorrhagic tie-2 mutants (Fig. 5Aiv) at every simvastatin concentration tested. Atorvastatin-induced hemorrhage occurred in the cranial region in wild-type fish (Fig. 5B), as well as in tie-2 mutants (Fig. 5B), and was detected by o-dianizidine staining in both genotypic groups (Fig. 5C). Our results strongly suggest that loss of Tie-2 protects from statin-induced bleeding.

To gain more insight into how atorvastatin causes bleeding in fish, we crossed tie-2 mutants to the $\operatorname{tg}(k d r-l: E G F P) s 8$ / $\operatorname{tg}($ gata1:dsRed)sd2 (Traver et al., 2003) double transgenic line. Time-lapse imaging of 1-day-old wild-type fish (Fig. 6Ai-v) revealed that atorvastatin causes vessel bursting: in $90 \%$ of cases the rupture in both wild type and mutants occurred during the formation of 

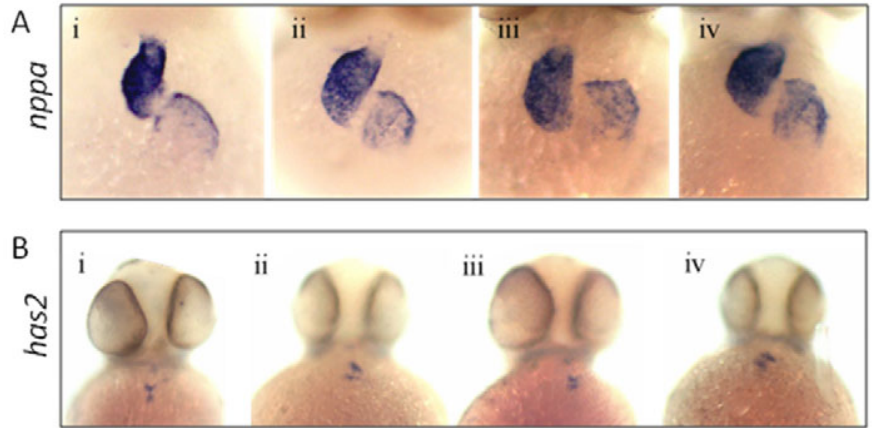

Fig. 3. Loss of Tie-1 and Tie-2 signaling does not affect nppa and has 2 expression. Whole-mount in situ hybridization for nppa (A) and has2 (B) in wild-type embryos (Ai; Bi), tie-2 mutants (Aii; Bii), wild-type embryos injected with sd-tie-1 MO (Aiii; Biii) and tie-2 mutants injected with sd-tie-1 MO (Aiv; Biv). Embryos were fixed at 48-50 hpf; all pictures were taken as ventral view.

the central arteries while branching from the primordial hindbrain channel at a time when a lumen was beginning to form, possibly suggesting a poor contact between the endothelial cells (Fig. 6Aiv,v) (supplementary material Movie 1). In the other $10 \%$ of cases the rupture occurred in vessels such as the optic artery, primordial midbrain channel and primitive prosencephalic artery. In tie-2 mutants the development of central arteries was completely normal, without vessel rupture and cranial hemorrhages (Fig. 6Bi-v) (supplementary material Movie 2). In the cases in which tie-2 mutants exhibited cranial hemorrhages, vessel rupture occurred during the formation of the central arteries while branching from the primordial hindbrain channel. In addition, we performed electron microscopy on non-exposed siblings (Fig. 6Avi) and mutants (Fig. 6Bvi) to investigate whether there was a difference in cell-cell junction number and/or morphology between the two groups. We were not able to observe any differences in junction number and morphology between wild-type siblings and mutants in the absence of atorvastatin. In atorvastatin-treated wild-type siblings and tie-2 mutants that developed hemorrhages, the lumen of the vessels in the head region was hardly recognizable (data not shown) and the endothelial cell-cell junctions could not be analyzed.

\section{tie-2 mutants are less resistant to atorvastatin-induced bleeding after VE-cadherin knockdown}

The observed effect of atorvastatin on vessel rupture in wild-type zebrafish suggests that atorvastatin affects endothelial cell permeability and cell-cell contacts in angiogenic sprouts. Therefore, we investigated whether knockdown of VE-cadherin, a major component of endothelial cell-cell adhesion complexes, affects the bleeding tendency in tie-2 mutants when exposed to atorvastatin. VE-cadherin has been reported to be crucial for vascular development (Carmeliet et al., 1999). Therefore, an appropriate morpholino dose was selected so that embryos developed normally, and no hemorrhagic phenotype was observed in $90 \%$ of the wildtype siblings and tie-2 mutants. We injected offspring from heterozygous mutant carriers with the previously validated $0.1 \mathrm{ng} / \mathrm{nl}$ $s d$-VE-cadherin MO (Nicoli et al., 2007), followed by exposure to different concentrations of atorvastatin (50, 150, 300, 500 and 1000 $\mathrm{nM}$ ) from $6 \mathrm{hpf}$ to $58 \mathrm{hpf}$. In parallel, offspring from heterozygous
A
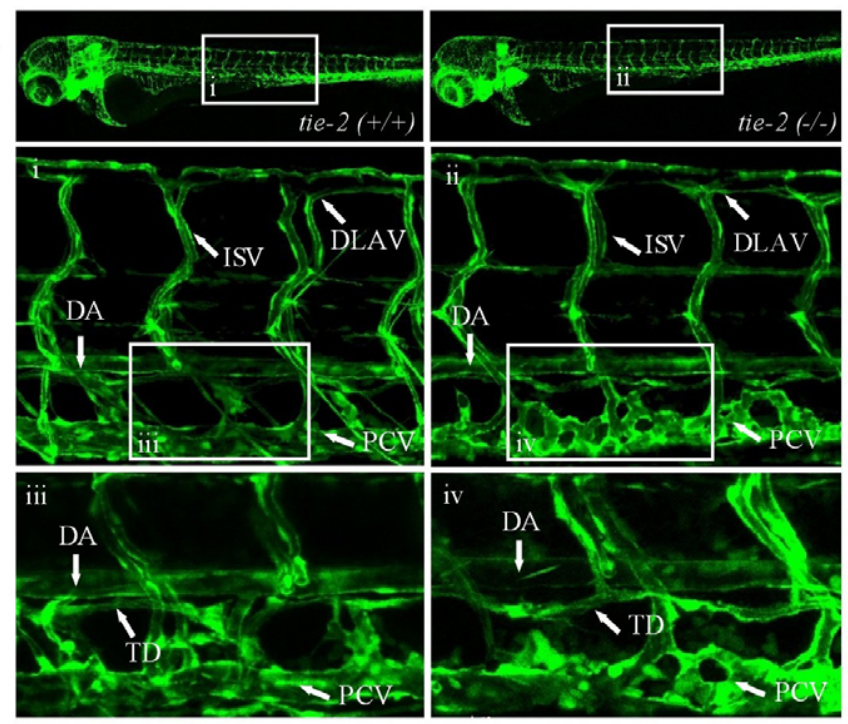

$\mathrm{B}$
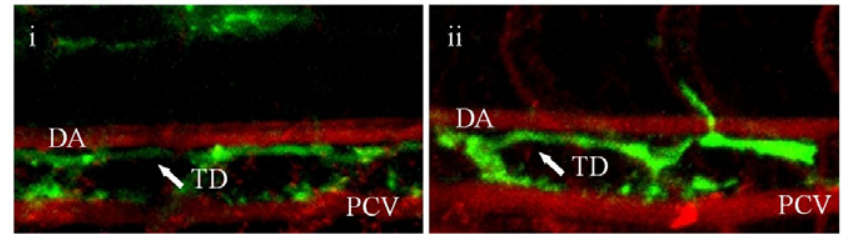

Fig. 4. tie-2 mutants develop normal blood and lymphatic vasculature. (A) Confocal microscopy of trunk vessels in 5-day-old wild-type (Ai) and tie-2 mutant (Aii) embryos in the $\mathrm{Tg}$ (fli1:EGFP) background. Loss of Tie-2 protein does not affect blood and lymphatic vessel formation (Aii,iv) in zebrafish as compared to the wild-type blood (Ai) and lymphatic (Aiii) vessel development. (B) A test for functionality of the thoracic duct shows dye uptake in both wildtype siblings $(\mathrm{Bi})$ and tie-2 mutants (Bii). Angiographies were performed in $\mathrm{Bi}$ and Bii to highlight the dorsal aorta (DA) and PCV. ISV, intersegmental vessels; TD, thoracic duct; DLAV, dorsal longitudinal anastomotic vessel.

mutant carriers were exposed to the same atorvastatin concentrations ( $n=50$ per concentration) as in the $s d$-VE-cadherinMO-injected group. Hemorrhages were again identified through o-dianizidine staining and both groups were genotyped after scoring. tie-2 homozygous mutants injected with $s d$-VE-cadherin MO displayed a significantly higher incidence of hemorrhaging than the non-injected mutants at all atorvastatin concentrations used (Fig. 7). The percentage of tie-2 homozygous mutant embryos that showed head hemorrhages after VE-cadherin knockdown was similar to the percentage of the siblings injected with $s d-V E$ cadherin MO for every atorvastatin concentration used. These results suggest that the vasoprotective effect in tie-2 mutants is dependent on normal levels of VE-cadherin.

\section{DISCUSSION}

We have identified a mutant that carries a stop mutation in the kinase domain of the zebrafish tie-2 gene. The stop mutation is Nterminal to Y1094 and Y1105 of the protein, two residues that correspond to Y1101 and Y1112 of murine Tie-2. These residues are conserved across species and have been shown to be important sites for auto-phosphorylation and association of downstream signaling molecules (Huang et al., 1995). Therefore, in conjunction 

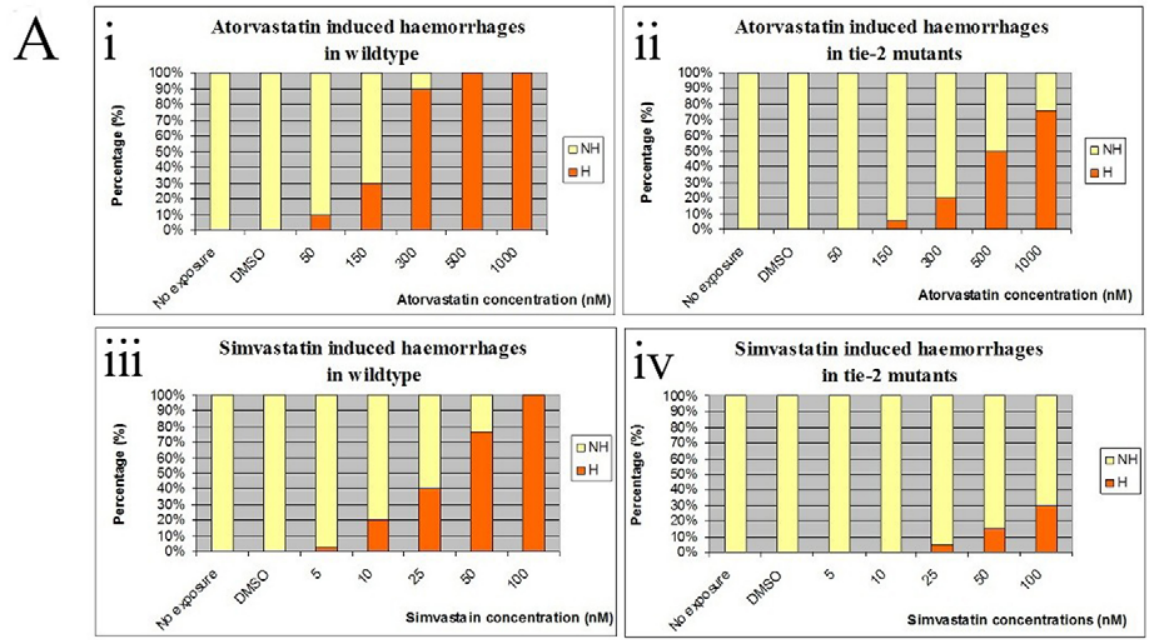

$\mathrm{B}$

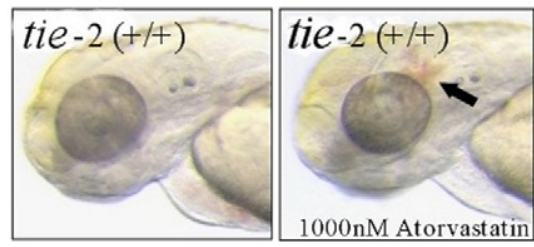

$\mathrm{C}$
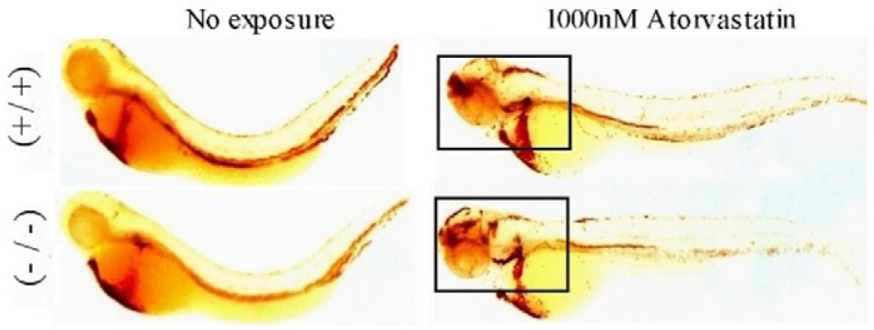
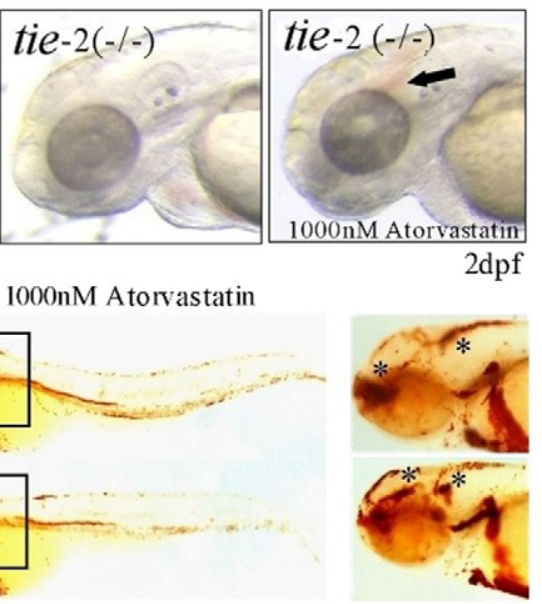

Fig. 5. Lack of Tie-2 protects zebrafish embryos from cranial hemorrhages caused by treatment with statins. (A) Graphic representation (in percentage) of hemorrhagic (orange bars) and nonhemorrhagic (yellow bars) groups for wild-type siblings (i) and tie-2 mutants (ii) after exposure to increasing concentrations of atorvastatin; the numbers of hemorrhagic (orange bars) and nonhemorrhagic (yellow bars) groups for wild-type siblings (iii) and tie-2 mutants (iv) after exposure to increasing concentrations of simvastatin are shown. (B) tie-2 mutants show similar cranial hemorrhages (arrow) as the wild-type embryos after treatment with atorvastatin. (C) No cranial hemorrhages are observed in wild-type siblings and tie-2 mutants as shown by odianizidine staining. However, treatment with 1000 $\mathrm{nM}$ atorvastatin causes cranial hemorrhages in the head region of both genotypic groups (asterisks show the regions in the head area of siblings and mutants where blood is accumulating). with the findings that tie-2 mRNA levels are severely reduced and immunohistochemistry using an anti-zebrafish antibody could not detect Tie-2 protein in 2-day-old mutants at all, we conclude that tie- $2^{\text {hu1667 }}$ represents a loss-of-function situation.

In contrast to the Tie-2 $2^{-/}$mouse phenotype, which is embryonic lethal mainly due to heart defects, $t i e-2^{-/-}$mutant fish are viable, show no defects during cardiac development, and undergo normal vasculogenesis, angiogenesis and lymphangiogenesis. However, knockdown of zebrafish tie-1 in the tie-2 mutant background shows phenotypes similar to those of the Tie-2-null mouse. This suggests that, in fish, there might be functional redundancy between the Tie- 2 and Tie- 1 proteins. Study of tie- 1 knockdown in fish suggests that tie-1 but not tie-2 is required in the early stages of heart development. However, both genes are crucial in later stages and show synergistic effects in the maintenance of endocardialmyocardial interaction in loss-of-function situations, a phenotype that is similar to the phenotype seen in Tie- $2^{-/}$mice, which have poor association between endothelial cells and the myocardium. This finding indicates that zebrafish Tie-1 and Tie- 2 function is to some extent different from the function of these receptors in mice. The most likely scenario is that an ancestral, single Tie gene was required for heart development and that, after genome duplication, functional divergence occurred within the vertebrate lineage: in fish, functional redundancy has been conserved between Tie- 1 and Tie-
2 in respect to cardiac development, whereas, in mice, Tie- 1 cannot substitute for a loss of Tie-2 function in the early heart.

Our finding that Tie-2 function in fish is not crucial during early stages of development offers the opportunity to study later roles of Tie- 2 in a systemic mutant situation in a vertebrate embryo, for example in the context of vessel stability. An important observation in the tie-2 mutants is their resistance to bleeding when exposed to atorvastatin. Atorvastatin reduces low-density lipoprotein cholesterol and is used in the clinic to lower the risk of stroke, heart attack or other heart complications in people with coronary heart disease or type 2 diabetes. Interestingly, one of the side effects of atorvastatin reported in patients is nose bleeding (http:// www.drugs.com/sfx/amlodipine-atorvastatin-side-effects.html). In addition, recent clinical studies have reported hemorrhagic stroke as a side effect of the atorvastatin treatment (Goldenstein et al., 2008; Vergouwen et al., 2008). Analysis of time-lapse microscopy in zebrafish reveals that atorvastatin, in $90 \%$ of cases, induces vessel rupture and bleedings in the region of the central arteries at the time when these vessels start to sprout from the primordial hindbrain channel. In $10 \%$ of cases, the vessel rupture happens in already-formed head vessels such as the primordial midbrain channel. The central arteries start to develop at 1.5 days postfertilization ( $\mathrm{dpf}$ ) and different processes such as sprouting, remodeling, migration and lumen formation take place 

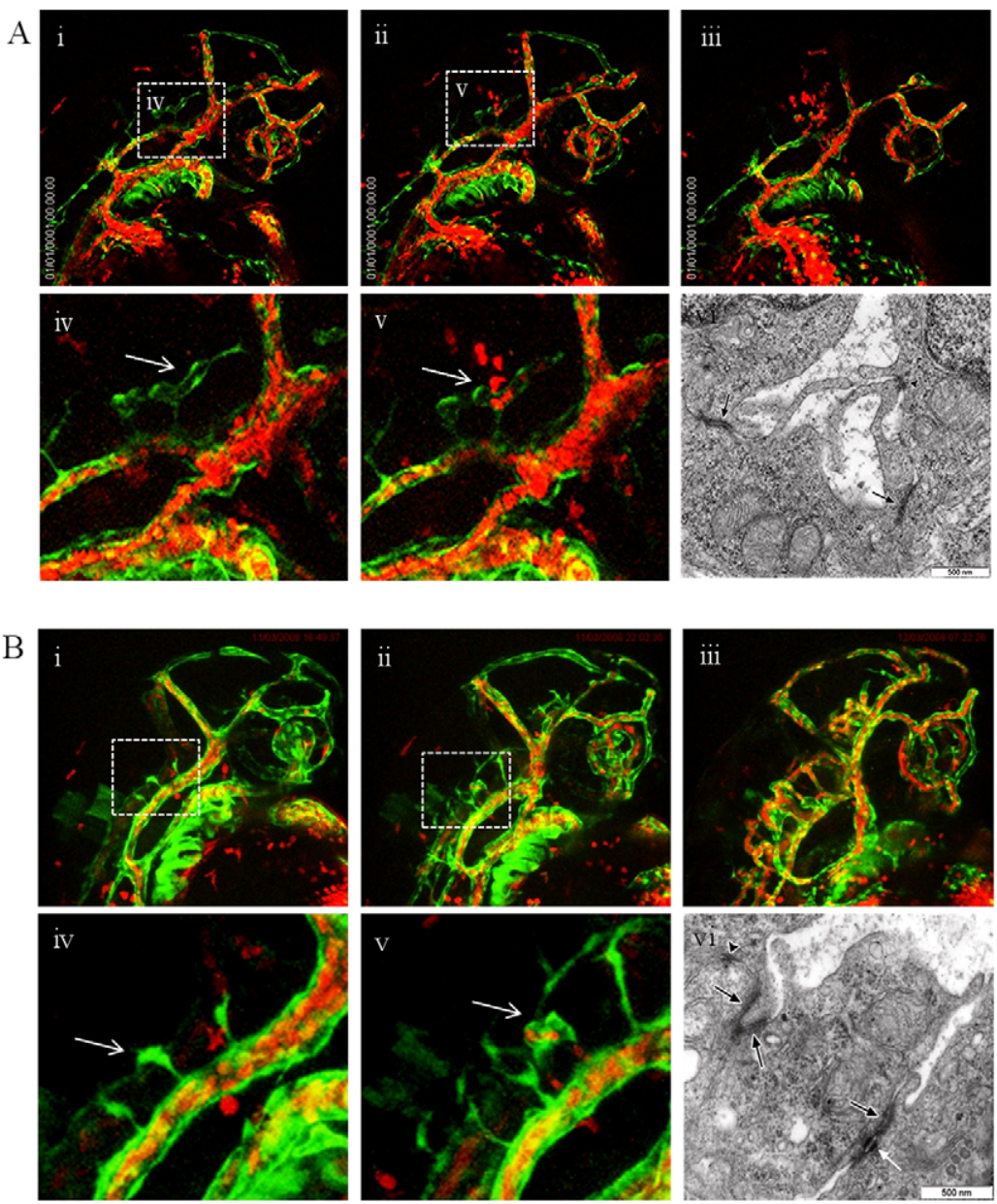

Fig. 6. Atorvastatin causes bleeding through vessel rupture. (A) Time-lapse imaging shows that atorvastatin causes vessel rupture and thus bleedings in siblings at the region of the central arteries (i-v). Ruptures occur precisely at the moment when blood enters the lumenized vessel (white arrow in iv and v). (vi) EM analysis in the head region of non-exposed siblings shows no defects in endothelial cell-cell contact. Black arrows indicate the junctional complex. (B) Time-lapse imaging shows that atorvastatin does not cause vessel rupture and thus bleedings in the tie-2 mutants at the region of the central arteries (i-v). No rupture occurs when blood enters the lumenized vessel (white arrow in iv and v). (vi) EM analysis in the head region of nonexposed tie-2 mutants shows no defects in endothelial cell-cell contact. Black arrows indicate the junctional complexes; white arrow indicates a junctional structure with desmosomal components; arrowhead indicates a junctional structure. Scale bars: $500 \mathrm{~nm}$. simultaneously. Detailed time-lapse analysis on wild-type fish and mutants shows that, even though there is apparent blebbing and endothelial plasticity occurring, the endothelial cells in tie-2 mutants stay intact and no vessel bursting occurs. Because we never observed endothelial cell death prior to the hemorrhages, this suggests that atorvastatin affects the endothelium through reducing endothelial cell-cell contacts prominently in newly forming vessels. VE-cadherin is one of the key regulators of cell-cell contacts in endothelial cells. Therefore, we investigated whether carefully titrated VE-cadherin knockdowns in tie-2 mutants affect the rate of hemorrhaging after treatment with atorvastatin. Interestingly, in these experimental settings we noticed that the percentage of tie-2 mutants that is affected by hemorrhages is similar to that of wild-type siblings. Therefore, our data show that VE-cadherin and Tie-2 might act in concert to allow vessel remodeling while maintaining vessel stability, respectively.

The finding that vessels in tie-2 mutants are more resistant to bleeding than in wild-type fish is somehow surprising because the ligand for Tie-2, Ang-1, has been reported to stabilize blood vessels and reduce permeability via different signaling pathways. Ang1-Tie-2 signaling prevents VEGF-induced endothelial permeability by activating $\mathrm{mDia}$ via $\mathrm{RhoA}$, which results in sequestering of Src through mDia. Because Src is therefore not able to initiate the phosphorylation of VE-cadherin, VE-cadherin is not internalized and remains in the inter-endothelial junctions, and thereby increases the barrier function of the endothelium. In 2007, Mammoto et al. provided a novel mechanism for the antipermeability effect of Ang-1-Tie-2 signaling and vessel permeability, showing that Ang-1 activates Rac1 by suppressing RhoA activity through p190RhoGAP to achieve its antipermeability effect (Mammoto et al., 2007). In their proposed mechanism, activation of Rac1 induces cortical actin fiber rearrangement and intensification of the junctional VE-cadherin, resulting in reduced permeability. Recently, Li et al. linked Ang1-mediated endothelial permeability with the regulation of sphingosine kinase-1 (SK-1), showing that Ang-1 inhibits vessel leaking by activating SK-1 (Li et al., 2008). Taken together, these data demonstrate that Ang-1 can regulate multiple pathways implicated in EC permeability, and cause different effects depending on the specific context. Therefore, one might assume that, in the absence of Tie- 2 signaling, vessels would be more destabilized and prone to leaking. This concept is supported by numerous reports on Ang-1-Tie-2 signaling and its role in endothelial permeability. These studies were carried out in mature quiescent vasculature (Suri et al., 1996; Thurston et al., 1999; Baffert et al., 2005; Hwang et al., 2009) and in mammalian cell culture systems (Gamble et al., 2007; Gavard et al., 2007; Milner et al., 2006). However, our data demonstrate that, under conditions 


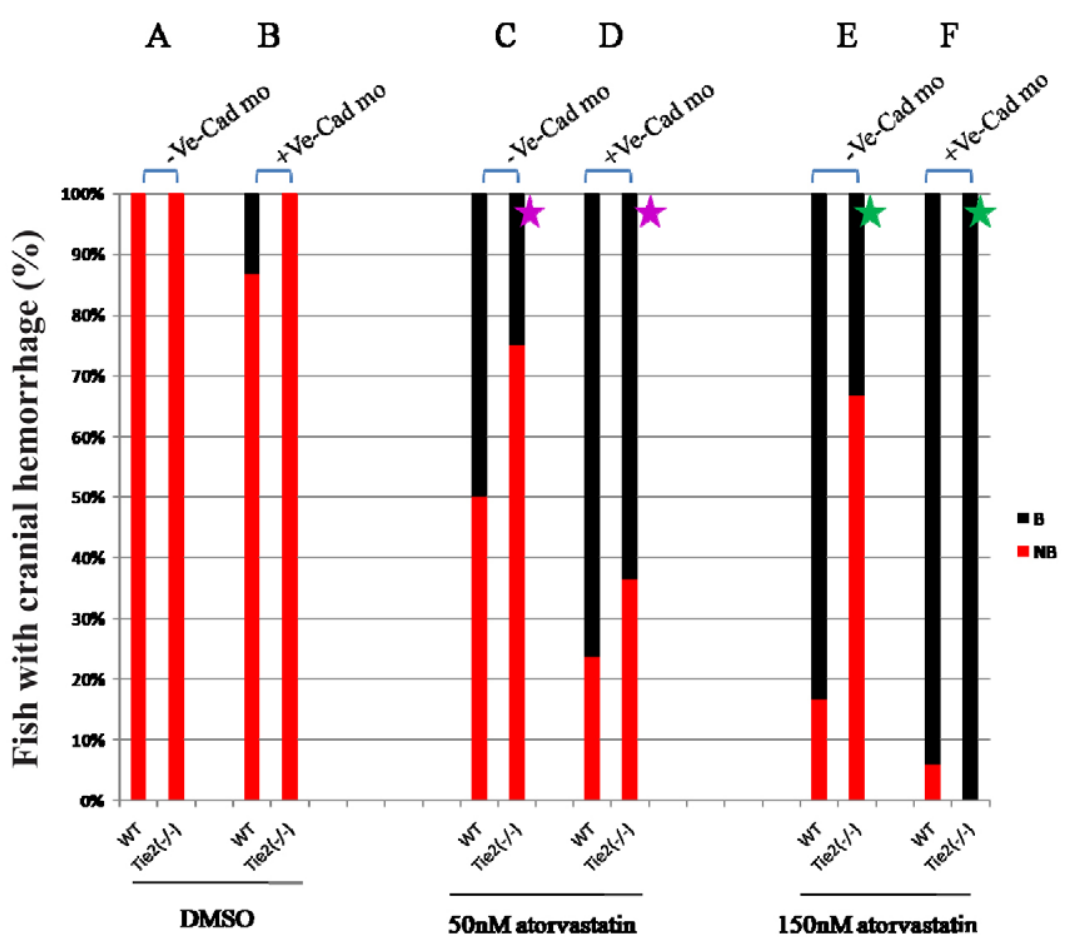

Fig. 7. Reduction in VE-cadherin levels in tie-2 mutants increases the rate of atorvastatin-induced bleeding. (A,B) VE-cadherin knockdown causes bleeding in $12 \%$ of wildtype siblings but has no effect in tie-2 mutants. (C) Exposure to $50 \mathrm{nM}$ atorvastatin results in cranial hemorrhages in noninjected wild-type siblings (50\%) and tie-2 mutants (24\%). (D) VE-cadherin knockdown further sensitizes wild-type siblings (77\%) and tie-2 mutants (63\%) to hemorrhaging when exposed to $50 \mathrm{nM}$ atorvastatin. Purple stars show the significance between non-injected and VE-cadherin-MOinjected tie-2 mutants $(P=0.03)$ calculated using paired Student's $t$-test. (E) Exposure to $150 \mathrm{nM}$ atorvastatin results in progressively more frequent cranial hemorrhages in noninjected siblings (83\%) and tie-2 mutants (32\%) compared with exposures with lower doses. (F) VE-cadherin knockdown increases the number of sibling (95\%) and tie-2 mutants (100\%) with hemorrhages when exposed to $150 \mathrm{nM}$ atorvastatin. Green stars show the significance between noninjected and VE-cadherin-MO-injected tie-2 mutants $(P=0.001)$ calculated using paired Student's $t$-test. Red bars (NB) represent non-hemorrhagic embryos and black bars (B) represent hemorrhagic embryos. using a particular agent to challenge vessel stability, lack of Tie-2 signaling can be protective in terms of vessel stability and integrity in zebrafish. We consider it unlikely that this apparent discrepancy might be explained by merely systemic differences between mammals and teleosts: given the conserved function of the Tie-1 and/or Tie-2 signaling axis in mammalian and zebrafish heart development, it seems unlikely that other aspects of Tie-2 function would diametrally diverge. Rather, we suggest that experimental conditions, the choice of in vivo versus in vitro system and the status of quiescent versus angiogenic vessels are of considerable importance when studying Tie-2 signaling.

In summary, our results show that Tie-1 and Tie- 2 have redundant roles in heart development in zebrafish and more specifically in the maintenance of endocardial-myocardial interaction. In this context it is of interest that some human VMCM patients that harbor p.R915H mutations suffer from ventricular septal defects, even though the hyper-phosphorylated state of the human mutant proteins might not be directly comparable to the loss-of-function situation of tie- 2 mutant zebrafish embryos. In addition, our data on tie- 2 mutants being protected from atorvastatin-induced cerebral hemorrhages suggests a role of Tie2 signaling in vessel permeability. The viability of tie- 2 mutant fish makes this a good model to study the Ang-1-Tie-2 signaling pathway, and its implication in vessel development and integrity. Furthermore, these mutants might allow screening for pharmacological compounds that alter Tie-2 function in vascular permeability.

\section{METHODS}

\section{Whole-mount in situ hybridization}

In situ hybridizations were performed essentially as described (Martyn and Schulte-Merker, 2004). Labeled probes were purified using NucleoSpin RNA clean-up columns (Macherey-Nagel). The substrate used in the second color reaction was [4-iodophenyl]-3[4-nitrophenyl]-5-phenyl-tetrazolium chloride (Roche). Previously described probes used were Tie-1 (Lyons et al., 1998), Tie-2 (Lyons et al., 1998), Anf (Smith et al., 2009) and Has2 (Smith et al., 2009).

\section{Zebrafish immunohistochemistry and o-dianizidine staining}

Immunohistochemistry on zebrafish sections using anti-Tie-2 (1:200, R\&D Systems) and anti-GFP (1:1000, Torrey Pines Biolabs) antibodies was performed as described (Dong et al., 2007). Secondary antibody used was Alexa-Fluor-488-anti-goat (1:1000, Invitrogen). Staining for erythrocytes was achieved by incubating embryos in a solution containing o-dianizidine (Sigma, St Louis, MO) as described (Paffet-Lugassy and Zon, 2004).

\section{MO-mediated knockdown of tie-1}

Antisense morpholino oligomers (Gene Tools, Corvalis, OR) for tie-1 (5'-AAAACCTGTACCTGTTTGTCACT-3') hybridize to the predicted exon-1-intron-1 junctions. MOs were resuspended in MilliQ water and $1 \mathrm{nl}$ was microinjected at the one-cell stage at an amount of 3, 6 and $8 \mathrm{ng}$ /embryo. sd-VE-cadherin MO (Nicoli et al., 2007), a kind gift from Marco Presta, University of Brescia, Italy, was injected at $0.1 \mathrm{ng} / \mathrm{embryo}$.

\section{Time-lapse analysis}

Embryos were mounted in 1\% low-melting agarose in a six-well culture plate with a cover slip on the bottom of the well and imaged with a Leica TCS SP confocal microscope using a $40 \times$ dry objective. Maximal $z$-projections of $40-50$ slices at $4 \mu \mathrm{m}$ per slice were compiled using ImageJ software. Time points were recorded every 5 minutes for 8-10 hours. A heated stage kept the temperature at approximately $28.5^{\circ} \mathrm{C}$. 


\section{TRANSLATIONAL IMPACT}

\section{Clinical issue}

Venous malformations are mainly of sporadic origin, but a small proportion, including glomuvenous malformations (OMIM \#138000) and cutaneomucosal venous malformations (VMCMs; OMIM \#600195), are inherited. VMCMs present as bluish patches mainly at the mucosa, and are caused by dilation of blood vessels. VMCMs have been genetically linked to endothelial-specific receptor tyrosine kinase 2 (TIE2; also known as TEK): several different mutations in this gene have been identified in VMCM patients. In line with the proposed roles of TIE2 in vascular remodeling and maintenance of mammalian vessel integrity, VMCM patients have a relative lack of mural cells in affected regions of the endothelium, and some VMCM patients have ventricular septal defects. Tie-2-1mice die during embryogenesis mainly owing to heart defects, providing clues about the role of TIE2 in the pathology of VMCM in humans. However, the precise functions of TIE2 in vascular stability and development are still not fully understood.

\section{Results}

In this study, the authors generate a zebrafish tie-2 loss-of-function mutant and show that, in contrast to the phenotype of Tie- $2^{-/-}$mice, tie- 2 mutant zebrafish are viable and show no defects in embryonic angiogenesis. However, morpholino-mediated knockdown of Tie-1 function in tie-2 zebrafish mutants shows that combined loss of Tie-1 and Tie-2 function results in defects of myocardial-endocardial contact. Given that a role has been proposed for Tie-2 in vascular permeability, the authors also challenge vessel stability in tie-2 mutants by using statins, which have been shown to induce hemorrhages in specific vascular regions of the zebrafish embryo. Surprisingly, tie-2 mutants are resistant to statin-induced bleeding, and further experiments indicate that this effect depends on VE cadherin, an important intercellular junction protein.

\section{Implications and future directions}

This work introduces an additional vertebrate model in which to study the function of Tie- 2 in vivo. Because Tie-1 and Tie-2 have redundant roles in cardiac development in zebrafish, this model will allow the study of Tie-2 function in vivo without the complication of cardiac defects. Tie- 2 is generally considered to be a vascular stabilizing factor, because it recruits support cells to the endothelium; however, the effect of statins in tie-2 mutants observed in this study suggest that the function of Tie-2 and/or its ligands might be more context-dependent than previously appreciated.

\section{doi:10.1242/dmm.006916}

\section{Electron microscopy fixation and preparation}

The fish were prepared for electron microscopy as described (De Maziere et al., 2008). Briefly, embryos were dechorionated and fixed with a mixture of $2 \%$ paraformaldehyde, $2.5 \%$ glutaraldehyde, $0.025 \% \mathrm{CaCl}_{2} \cdot 2 \mathrm{H}_{2} \mathrm{O}$ and $0.1 \mathrm{M} \mathrm{Na}$-cacodylate, $\mathrm{pH}$ 7.4. After rinsing in $0.1 \mathrm{M} \mathrm{Na}$-cacodylate at $4^{\circ} \mathrm{C}$, the embryos were postfixed with $2 \% \mathrm{OsO}_{4}$ for 2 hours at room temperature, stained and blocked with $0.5 \%$ uranyl acetate, dehydrated with acetone and embedded in Epon. Ultrathin sections were stained with uranyl acetate in $70 \%$ methanol and Reynold's lead citrate. Analysis was done at $80 \mathrm{kV}$ using a Tecnai 12 (FEI Company, Eindhoven, The Netherlands) equipped with a side-mounted MegaView II camera (SIS, Münster, Germany).

\section{ACKNOWLEDGEMENTS}

We thank Ann Maziere for help with zebrafish electron microscopy analysis, Jeroen Kuipers for his expertise and genuine help with time-lapse confocal microscopy, Josi Peterson-Maduro for help with molecular cloning techniques, Harry Begthel for advice on zebrafish immunohistochemistry experiments, and Marco Presta for providing the VE-cadherin morpholino. We also thank Stephan Huveneers, Johan de Rooij, Ben Hogan, Terhi Karpanen and Jeroen Bussmann for valuable discussions.

\section{COMPETING INTERESTS}

The authors declare no competing financial interests.

\section{AUTHOR CONTRIBUTIONS}

E.G., A.K., E.W. and S.S.-M. designed the experiments. E.G., P.S. and L.H.H. performed the experiments. E.G., P.S., J.-A.P., K.A., L.H.H. and S.S.-M. analyzed the data. E.G. and S.S.-M. wrote the manuscript.

\section{SUPPLEMENTARY MATERIAL}

Supplementary material for this article is available at http://dmm.biologists.org/lookup/suppl/doi:10.1242/dmm.005033/-/DC1

\section{REFERENCES}

Baffert, F., Le, T., Thurston, G. and McDonald, D. M. (2005). Angiopoietin-1 decreases plasma leakage by reducing number and size of endothelial gaps in venules. Am. J. Physiol. Heart Circ. Physiol. 290, 107-118.

Bussmann, J., Bos, F., Urasaki, A., Kawakami, K., Duckers, H. J. and Schulte-Merker S. (2010). Arteries provide essential guidance cues for lymphatic endothelial cells in the zebrafish trunk. Development 137, 253-257.

Carmeliet, P., Lampugnani, M. G., Moons, L., Breviario, F., Compernolle, V., Bono, F., Balconi, G., Spagnuolo, R., Oosthuyse, B., Dewerchin, M. et al. (1999). Targeted deficiency or cytosolic truncation of the VE-cadherin gene in mice impairs VEGFmediated endothelial survival and angiogenesis. Cell 98, 147-157.

De Mazière, A., Parker, L., Van Dijk, S., Ye, W. and Klumperman, J. (2008). Egfl7 knockdown causes defects in the extension and junctional arrangements of endothelial cells during zebrafish vasculogenesis. Dev. Dyn. 237, 580-591.

Dong, P. D., Munson, C. A., Norton, W., Crosnier, C., Pan, X., Gong, Z., Neumann, C. J. and Stainier, D. Y. (2007). Fgf10 regulates hepatopancreatic ductal system patterning and differentiation. Nat. Genet. 39, 397-402.

Dumont, D. J., Gradwohl, G., Fong, G. H., Puri, M. C., Gertsenstein, M., Auerbach, A. and Breitman, M. L. (1994). Dominant-negative and targeted null mutations in the endothelial receptor tyrosine kinase, tek, reveal a critical role in vasculogenesis of the embryo. Genes. Dev. 8, 1897-1909.

Dumont, D. J., Fong, G. H., Puri, M. C., Gradwohl, G., Alitalo, K. and Breitman, M. L. (1995). Vascularization of the Mouse embryo: a study of flk-1, tek, tie, and vascular endothelial growth factor expression during development. Dev. Dyn. 203, 80-92.

Fiedler, U., Scharpfenecker, M., Koidl, S., Hegen, A., Grunow, V., Schmidt, J. M., Kriz, W., Thurston, G. and Augustin, H. G. (2004). The Tie-2 ligand angiopoietin-2 is stored in and rapidly released upon stimulation from endothelial cell Weibel-Palade bodies. Blood 103, 4150-4156.

Fiedler, U., Reiss, Y., Scharpfenecker, M., Grunow, V., Koidl, S., Thurston, G., Gale, N. W., Witzenrath, M., Rosseau, S. and Suttorp, N. (2006). Angiopoietin-2 sensitizes endothelial cells to TNF-alpha and has a crucial role in the induction of inflammation. Nat. Med. 12, 235-239.

Fukuhara, S., Sako, K., Noda, K., Nagao, K., Miura, K. and Mochizuki, N. (2008). Tie2 is tied at the cell-cell contacts and to extracellular matrix by Angiopoietin-1. Cell Biol. 10, 513-526.

Gale, N. W., Thurston, G., Hackett, S. F., Renard, R., Wang, Q., McClain, J., Martin, C., Witte, C., Witte, M. H., Jackson, D. et al. (2002). Angiopoietin-2 is required for postnatal angiogenesis and lymphatic patterning, and only the later role is rescued by Angiopoietin-1. Dev. Cell 3, 411-423.

Gamble, J. R., Drew, J., Trezise, L., Underwood, A., Parsons, M., Kasminkas, L., Rudge, J., Yancopoulos, G. D. and Vadas, M. A. (2007). Angiopoietin-1 is an antipermeability and anti-inflammatory agent in vitro and targets cell junctions. Circ. Res. 87, 603-607.

Gavard, J., Patel, V. and Gutkind, J. S. (2007). Angiopoietin-1 prevents VEGF-induced endothelial permeability by sequestering Src through mDia. Dev. Cell 14, 25-36.

Geva, E. and Jaffe, R. B. (2000). Role of angiopoietins in reproductive tract angiogenesis. Obstet. Gynecol. Surv. 55, 511-519.

Goldenstein, L. B., Amarenco, P., Szarek, A., Callahan, I. I. I., Hennerici, M., Sillesen, H., Zivin, J. A. and Welch, K. M. A. (2008). Hemorrhagic stroke in the Stroke Prevention by Aggressive Reduction in Cholesterol Levels study. Neurology 70, 2364 2370.

Hogan, B. M., Bussmann, J., Wolburg, H. and Schulte-Merker, S. (2008). ccm1 cell autonomously regulates endothelial cellular morphogenesis and vascular tubulogenesis in zebrafish. Hum. Mol. Genet. 17, 2424-2432.

Huang, L., Turck, C. W., Rao, P. and Peters, K. G. (1995). GRB2 and SH-PTP2: potentially important endothelial signaling molecules downstream of the TEK receptor tyrosine kinase. Oncogene 1, 1-7.

Hwang, J. A., Lee, E. H., Lee, S. D., Park, J. B., Jeon, B. H. and Cho, C. H. (2009) COMP-Ang1 ameliorates leukocyte adhesion and reinforces endothelial tight junctions during endotoxemia. Biochem. Biophys. Res. Commun. 381, 592-596.

Iwama, A., Hamaguchi, I., Hashiyama, M., Murayama, Y., Yasunaga, K. and Suda, T. (1993). Molecular cloning and characterization of mouse tie and tek receptor tyrosine kinase genes and their expression in hematopoietic stem cells. Biochem. Biophys. Res. Commun. 195, 301-309. 
Jin, S. W., Beis, D., Mitchell, T., Chen, J. N. and Stainier, D. Y. R. (2005). Cellular and molecular analyses of vascular tube and lumen formation in zebrafish. Development 132, 5199-5209.

Jin, S. W., Herzog, W., Santoro, M. M., Mitchell, T. S., Frantsve, J., Jungblut, B., Beis, D., Scott, I. C., D'Amico, L. A., Ober, E. A. et al. (2007). A transgene-assisted genetic screen identifies essential regulators of vascular development in vertebrate embryos. Dev. Biol. 307, 29-42.

Küchler, A. (2004). Die Rolle der Rezeptortyrosinkinase Tie2 bei der Gefässentwicklung des Zebrafisches, Danio rerio. PhD thesis, Universität Tübingen, Tübingen, Germany.

Lawson, N. D., Vogel, A. M. and Weinstein, B. M. (2002). Sonic Hedgehog and vascular endothelial growth factor acts upstream of the Notch pathway during arterial endothelial differentiation. Dev. Cell 3, 127-136.

Li, X., Stankovic, M., Bonder, C. S., Hahn, C. N., Parsons, M., Pitson, S. M., Xia, P. Proia, R. L., Vadas, M. A. and Gamble, J. R. (2008). Basal and angiopoietin-1mediated endothelial permeability is regulated by sphingosine kinase-1. Blood 111, 3489-3497.

Lyons, M. S., Bell, B., Stainier, D. and Peters, K. G. (1998). Isolation of the zebrafish homologues for the tie-1 and tie-2 endothelium-specific receptor tyrosine kinases. Dev. Dyn. 212, 133-140.

Maisonpierre, P. C., Suri, C., Jones, P. F., Bartunkova, S., Wiegand, S. J., Radziejewski, C., Compton, D., McClain, J., Aldrich, T. H., Papadopoulos, N. et al. (1997). Angiopoietin-2, a natural antagonist for Tie2 that disrupts in vivo angiogenesis. Science 277, 55-60.

Mammoto, T., Parikh, S. M., Mammoto, A., Gallagher, D., Chan, B., Mostoslavsky, G., Ingber, D. E. and Sukhatme, V. P. (2007). Angiopoietin-1 requires p190 RhoGAP to protect against vascular leakage in vivo. J. Biol. Chem. 282, 23910-23918.

Martyn, U. and Schulte-Merker, S. (2004). Zebrafish neuropilins are differentially expressed and interact with vascular endothelial growth factor during embryonic development. Dev. Dyn. 231, 33-42.

Milner, C. S., Hansen, T. M., Singh, H. and Brindle, N. P. (2006). Roles of the receptor tyrosine kinases Tie1 and Tie2 in mediating the effects of angiopoietin-1 on endothelial permeability and apoptosis. Microvasc. Res. 77, 187-191.

Nicoli, S., Ribatti, D., Cotelli, F. and Presta, M. (2007). Mammalian tumor xenografts induce neovascularization in zebrafish embryos. Cancer Res. 67, 2927-2931.

Paffett-Lugassy, N. N. and Zon, L. I. (2004). Analysis of hematopoietic development in the zebrafish. Methods Mol. Med. 105, 171-198.

Patan, S. (1998). TIE1 and TIE2 receptor tyrosine kinases inversely regulate embryonic angiogenesis by the mechanism of intussusceptive microvascular growth. Microvasc Res. 56, 1-21.

Puri, M. C., Rossant, J., Alitalo, K., Bernstein, A. and Partanen, J. (1995). The receptor tyrosine kinase TIE is required for integrity and survival of vascular endothelial cells. EMBO J. 14, 5884-5891.

Puri, M. C., Partanen, J., Rossant, J. and Bernstein, A. (1999). Interaction of the TEK and TIE receptor tyrosine kinases during cardiovascular development. Development 126, 4569-4580.

Saharinen, P., Kerkela, K., Ekman, N., Marron, M., Brindle, N., Lee, G. M., Augustin, H., Koh, G. Y. and Alitalo, K. (2005). Multiple angiopoietin recombinant proteins activate the Tie1 receptor tyrosine kinase and promote its interaction with Tie2. J. Cell Biol. 169, 239-243.

Saharinen, P., Eklund, L., Miettinen, J., Wirkkala, R., Anisimov, A., Winderlich, M., Nottebaum, A., Vestwebe, D., Deutsch, U., Koh, G. Y. et al. (2008). Angiopoietins assemble distinct Tie2 signalling complexes in endothelial cell-cell and cell-matrix contacts. Nat. Cell Biol. 10, 527-537.

Sato, T. N., Qin, Y., Kozak, C. A. and Audus, K. L. (1993). Tie-1 and Tie-2 define another class of putative receptor tyrosine kinase genes expressed in early embryonic vascular system. Prot. Natl. Acad. Sci. USA 90, 9355-9358.

Sato, T. N., Tozawa, Y., Deutsch, U., Wolburg-Buchholz, K., Fujiwara, Y., GendronMaguire, M., Gridley, T., Wolburg, H., Risau, W. and Qin, Y. (1995). Distinct roles of the receptor tyrosine kinases Tie-1 and Tie-2 in blood vessel formation. Nature $\mathbf{3 7 6}$, 70-74.

Smith, K. A., Joziasse, I. C., Chocron, S., van Dinther. M., Guryev, V., Verhoeven, M. C., Rehmann, H., van der Smagt, J. J., Doevendans, P. A., Cuppen, E. et al. (2009) Dominant-negative ALK2 allele associates with congenital heart defects. Circulation 119, 3062-3069.

Suri, C., Jones, P. F., Patan, S., Bartunkova, S., Maisonpierre, P. C., Davis, S., Sato, T. N. and Yancopoulos, G. D. (1996). Requisite role of Angiopoietin-1, a ligand for the TIE2 receptor, during embryonic angiogenesis. Cell 87, 1161-1169.

Suri, C., McClain, J., Thurston, G., McDonald, D. M., Zhou, H., Oldmixon, E. H., Sato, T. N. and Yancopoulos, G. D. (1998). Increased vascularization in mice overexpressing angiopoietin-1. Science 282, 468-471.

Teichert-Kuliszewska, K., Maisonpierre, P. C., Jones, N., Campbell, A. I., Master, Z., Bendeck, M. P., Alitalo, K., Dumont, D. J., Yancopoulos, G. D. and Stewart, D. J. (2001). Biological action of angiopoietin-2 in a fibrin matrix model of angiogenesis is associated with activation of Tie2. Cardiovasc. Res. 49, 659-670.

Thurston, G., Suri, C., Smith, K., McClain, J., Sato, T. N., Yancopoulos, G. D. and McDonald, D. M. (1999). Leakage resistant blood vessels in mice transgenically overexpressing angiopoietin-1. Science 286, 2511-2514.

Thurston, G., Rudge, J. S., loffe, E., Zhou, H., Ross, L., Croll, S. D., Glazer, N., Holash, J., McDonald, D. M. and Yancopoulos, G. D. (2000). Angiopoietin-1 protects the adult vasculature against plasma leakage. Nat. Med. 6, 460-463.

Traver, D., Paw, B. H., Poss, K., Penberthy, W. T., Lin, S. and Zon, L. I. (2003). Transplantation and in vivo imaging of multilineage engraftment in zebrafish bloodless mutants. Nat. Immunol. 4, 1238-1246.

Vergouwen, M. D., de Haan, R. J., Vermeulen, M. and Roos, Y. B. (2008). Statin treatment and the occurrence of hemorrhagic stroke in patients with a history of cerebrovascular disease. Stroke 39, 497-502.

Vikkula, M., Boon, L. M., Carraway, K. L., 3rd, Calvert, J. T., Diamonti, A. J., Goumnerov, B., Pasyk, K. A., Marchuk, D. A., Warman, M. L., Cantley, L. C. et al. (1996). Vascular dysmorphogenesis caused by an activating mutation in the receptor tyrosine kinase TIE2. Cell 87, 1181-1190.

Wienholds, E., Schulte-Merker, S., Walderich, B. and Plasterk, R. H. A. (2002). Target-selected inactivation of the zebrafish rag1 gene. Science 297, 99-102.

Wouters, V., Limaye, N., Uebelhoer, M., Irrthum, A., Boon, L. M., Mulliken, J. B., Enjolras, O., Baselga, E., Berg, J., Dompmartin, A. et al. (2010). Hereditary cutaneomucosal venous malformations are caused by TIE2 mutations with widely variable hyper-phosphorylating effects. Eur. J. Hum. Genet. 18, 414-420.

Yancopoulos, G. D., Davis, S., Gale, N. W., Rudge, J. S., Wiegand, S. J. and Holash, J. (2000). Vascular-specific growth factors and blood vessel formation. Nature 407, 242248

Yano, M., Iwama, A., Nishio, H., Suda, J., Takada, G. and Suda, T. (1997). Expression and function of murine receptor tyrosine kinases, Tie and Tek, in hematopoietic stemm cells. Blood 89, 4317-4326. 